\title{
Utilização do método Trigger Tool para identificação de reações adversas a medicamentos em um hospital privado de Cascavel - PR
}

\author{
Use of a Trigger Tool to detect adverse drug reactions in a private hospital in Cascavel - PR \\ Utilización del método Trigger Tool para la identificación de reacciones adversas a los \\ medicamentos en un hospital privado en Cascavel - PR
}

Recebido: 03/10/2021 | Revisado: 09/10/2021 | Aceito: 14/10/2021 | Publicado: 17/10/2021

\author{
Ana Flávia Lino Francisconi \\ ORCID: https://orcid.org/0000-0002-8343-2911 \\ Centro Universitário da Fundação Assis Gurgacz, Brasil \\ Email: aflfrancisconi@minha.fag.edu.br \\ Juliana Bordignon \\ ORCID: https://orcid.org/0000-0001-9766-736X \\ Centro Universitário da Fundação Assis Gurgacz, Brasil \\ E-mail: jubordig@gmail.com \\ Vagner Fagnani Linartevichi \\ ORCID: https://orcid.org/0000-0002-2624-7744 \\ Centro Universitário da Fundação Assis Gurgacz, Brasil \\ E-mail: linartevichi@fag.edu.br
}

\begin{abstract}
Resumo
A farmacovigilância é uma das responsabilidades do farmacêutico clínico, sendo que a implantação do método de identificação de gatilhos é uma maneira de evitar riscos à saúde do paciente e custos desnecessários ao sistema. Neste sentido, o presente trabalho tem como objetivo analisar a eficiência da farmacovigilância por meio do método Trigger Tool, implantado em um hospital privado de Cascavel, analisando ocorrência das principais reações adversas à medicamentos. A metodologia adotada para a elaboração deste material foi o estudo transversal retrospectivo, com coleta de dados por meio de relatórios manuais e também gerados pelo software Tasy. Ao final do estudo, foram identificadas 39 suspeitas de RAM, no qual a população estudada fora classificada como adultos com idade média 50,7 anos, com predominância do sexo feminino. O principal gatilho identificado foi o Metronidazol (16), seguido de Prometazina (13) e Hidrocortisona (10). Concluiu-se que o uso dos rastreadores se mostrou útil para a investigação, notificação e registro nos prontuários dos pacientes, ficando acessível para consultas e possíveis internamentos posteriores. Embora não possibilite a identificação da causa das RAM por conta da alta complexibilidade da farmacoterapia dos pacientes estudados.
\end{abstract}

Palavras-chave: Farmacovigilância; Efeitos colaterais e reações adversas relacionados a medicamentos; Segurança do paciente.

\begin{abstract}
Pharmacovigilance is one of the responsibilities of the clinical pharmacist and the implementation of the trigger identification method is a way to avoid risks to the patient's health and some unnecessary costs to the system. In this sense, this study aims to analyze the efficiency of pharmacovigilance through the "trigger tool" method implemented in a private hospital in the city of Cascavel, Paraná - Brazil, and the main adverse drug reactions that occurred. The methodology adopted was to carry out a retrospective cross-sectional study, with data collection through manual reports and also generated by the Tazy program. At the end of the study, 39 ADR were identified, in which the population studied was classified as adults with a mean age of 50.7 years, with a predominance of females. The main trigger identified was Metronidazole (16), followed by Promethazine (13) and Hydrocortisone (10). It was concluded that the use of trackers proved to be useful for investigation, notification and registration in patients' medical records, making it accessible for consultations and possible subsequent hospitalizations, although it does not allow the identification of the cause of ADR due to the high complexity of the pharmacotherapy of the studied patients.
\end{abstract}

Keywords: Pharmacovigilance; Drug-related side effects and adverse reactions; Patient safety.

\section{Resumen}

La farmacovigilancia es una de las responsabilidades del farmacéutico clínico, siendo que la implantación del método de gatillos es una manera de evitar riesgos a la salud del paciente y costos innecesarios al sistema. En este sentido, el presente trabajo tiene como objetivo analizar la eficiencia de la farmacovigilancia por medio del método Trigger Tools, implantado en un hospital privado de Cascavel, analizando ocurrencias de las principales reacciones adversas a 
los medicamentos. La metodología adoptada para la elaboración de este material fue el estudio transversal retrospectivo, con colectas por medio de informes mensuales y también generados por el software Tasy. Al final del estudio, fueran identificadas 39 RAM, en las cuales la población estudiada fuera clasificada como adultos con media de edad de 50,7 años, con predominancia del sexo femenino. El principal gatillo identificado fue el Metronidazol (16), seguido de Prometazina (16) e Hidrocortisona (10). En conclusión, el uso de los rastreadores se mostró útil para la investigación, notificación y registro en los historiales médicos de los pacientes, estando accesible para consultas y posibles internaciones posteriores. Aunque no posibilita la identificación de la causa de las RAM causa de la alta complejidad de la farmacoterapia de los pacientes estudiados.

Palabras clave: Farmacovigilancia; Efectos colaterales y reacciones adversas relacionados con medicamentos; Seguridad del paciente.

\section{Introdução}

Devido a ampla farmacoterapia pacientes internados apresentam risco elevado para reações adversas a medicamentos (RAM). Neste contexto, é visto a importância de cuidados realizados por uma equipe multiprofissional que esteja preparada para identificar sinais e sintomas que sejam indicativos de efeitos adversos a medicamentos, a fim de reduzir possíveis danos ao paciente (OPAS, 2002).

A Organização Mundial de Saúde (OMS, 2005) define reação adversa a medicamento (RAM), como qualquer resposta prejudicial ou indesejável e não intencional que ocorre com medicamentos em doses normalmente utilizadas para profilaxia, diagnóstico, tratamento de doença ou para modificação de funções fisiológicas (Minella \& Linartevichi, 2021). Sendo que diversos fatores estão relacionados com o desenvolvimento de reações adversas a medicamentos, incluindo, idade avançada, estado clínico severo, comorbidades e a ampla farmacoterapia (Sousa et al., 2020).

Desta forma, além do risco aumentado em pacientes já hospitalizados, as RAM's demonstram também uma alta na demanda de serviços de saúde e consequentemente gastos adicionais. Para reduzir tais problemas deve-se tomar conhecimento da real incidência e classificá-las a fim de garantir mais segurança ao paciente e reduzir custo ao sistema (Souza et al., 2018).

Neste contexto vê-se a importância do profissional farmacêutico clinico e a implementação da farmacovigilância, a qual tem como principal objetivo a promoção do uso racional de medicamentos a fim de obter aproveitamento máximo terapêutico e evitar efeitos indesejáveis (CFF, 2013). Visto isso, diversos programas para detecção e identificação de reações adversas foram criados por organizações de saúde, dentre eles, o método Trigger tool. Esse método consiste na busca retrospectiva nos prontuários dos pacientes, utilizando de dados considerados "gatilhos" para reações adversas (Araujo et al., 2018).

Nessa metodologia a presença de gatilhos tratam-se de indicativos ou evidências registradas em prontuário que apontam a possibilidade de que tenha ocorrido algum evento adverso durante o tratamento do paciente, eventos como a prescrição de fármacos específicos, exames com valores anormais, suspensões abruptas de medicamentos, são utilizados como gatilhos para a pesquisa dos prontuários, se um gatilho for identificado em um registro, indica apenas a presença de um gatilho, não necessariamente um evento adverso. O revisor deve investigar os detalhes para determinar se um evento adverso realmente ocorreu (Araujo et al., 2019).

Para aumentar a eficiência do processo de avaliação de causa e padronizar os eventos adversos diversos métodos tem sido desenvolvidos. Os algoritmos são métodos sistemáticos em forma de questionários onde são considerados alguns critérios para avaliação das RAMs (Filho, 2014). No hospital em estudo, o algoritmo utilizado é o de Liverpool ADR causality tool Liverpool, o qual é composto por perguntas com respostas sim e não, em forma de um fluxograma, que leva a decisão se a RAM é definida, provável, possível ou improvável (Souza, 2016).

Além disso, as identificações das RAM são feitas através de busca ativa, que de acordo com dados trata-se da maneira mais eficiente de rastreio. Entretanto, demanda tempo, recursos humanos e financeiros para ser bem empregado. Bem como, a eficácia do método, quando realizado retroativa, depende da qualidade dos registros dos profissionais, o que também se torna 
um fator limitante (Sousa et al., 2021).

O presente trabalho, teve como principal objetivo analisar a eficiência da farmacovigilância por meio do método Trigger Tool adaptado a realidade do hospital, a fim de verificar as principais suspeitas de reações adversas que ocorreram, suas frequências, perfis e o desfecho clínico dos pacientes priorizando a segurança e efetividade do tratamento.

\section{Metodologia}

Trata-se de um estudo transversal, com coleta de dados retrospectivo. Realizado em um hospital privado de médio porte, localizado no município de Cascavel - Pr e aprovado pelo comitê de ética sob CAAE nº 47206721.3.0000.5219. Onde foram estudados pacientes com idade igual e superior a 18 anos de todas as alas do hospital. O período de estudo foi de janeiro a julho de 2021.

As identificações das reações adversas foram realizadas a partir de uma busca ativa, através de uma lista de medicamentos gatilhos, conforme exposto pela Tabela 1, pré-estipulada pela própria farmácia clínica do hospital e adaptado de Carvalho et al. (2017). Todos os usuários destes medicamentos, no período estudado, foram avaliados pelo profissional farmacêutico.

Tabela 1. Medicamentos gatilho.

\begin{tabular}{|c|c|}
\hline Medicamento & Suspeita de RAM ou motivo do uso \\
\hline Naloxona $\left(\operatorname{Narcan}^{\circledR}\right)$ & Sedação causada por opioides \\
\hline Flumazenil (Lanexat ${ }^{\circledR}$ ) & Sedação causada por benzodiazepínicos \\
\hline Metronidazol VO $\left(\right.$ Flagyl $^{\circledR}$ ) & Diarreia causada pelo uso de Antibióticos \\
\hline $\begin{array}{l}\text { Anti-histamínicos: Dexclorfeniramina }\left(\text { Polaramine }{ }^{\circledR}\right), \\
\left.\text { Betametasona }\left(\text { Celestamine }^{\circledR}\right), \text { Fexofenadina }\left(\text { Allegra }^{\circledR}\right) \text {, Hidroxizina } \text { (Hixizine }^{\circledR}\right) \text {, } \\
\text { Prometazina }\left(\text { Fenergan }{ }^{\circledR}\right) \text {, } \\
\text { Corticoides: Hidrocortisona }\left(\text { Solu-cortef }{ }^{\circledR}\right)\end{array}$ & Potenciais indicadores de reação alérgica \\
\hline
\end{tabular}

Fonte: Adaptado de Carvalho, et al. (2017).

Semanalmente, eram emitidos os relatórios de uso dos medicamentos gatilhos através do sistema Tasy e realizado a busca de sinais e sintomas que sugerissem a possibilidade de RAM's. Em seguida, por meio do prontuário eletrônico, cada caso foi analisado e os considerados suspeitos foram definidos como possíveis RAMs e avaliado por meio da ferramenta de Liverpool sua possível causalidade (Gallagher et al., 2011). Além da identificação dos gatilhos, sempre que foi identificado o uso de Metronidazol administrado por via oral era também investigado se houve a solicitação de exames laboratoriais para pesquisa de Clostridium difficile ou suas toxinas e também se o paciente estava fazendo uso de dietas ou medicamentos via sondas enterais que podem ser causadores de diarreia assim como o uso de alguns antimicrobianos (Almeida \& Genaro, 2019). Após a identificação da possível causa, os dados foram tabelados e posteriormente realizada notificação ao Núcleo de Segurança do Paciente (NSP), de maneira que tal reação fosse inclusa no prontuário do paciente a fim de evitar recorrência.

\section{Resultados e Discussão}

A população estudada foi classificada como adultos, com idade média de 50,7 anos, sendo a idade mínima de 18 e máxima de 87, conforme demonstração na Tabela 2. 
Tabela 2. Faixa etária dos pacientes que apresentaram reações adversas identificadas no município de Cascavel - Pr, 2021.

\begin{tabular}{ll}
\hline Faixa etária & $\mathbf{N}(\boldsymbol{\%})$ \\
\hline $18-39$ & $14(36)$ \\
$40-59$ & $11(28)$ \\
$60-89$ & $14(36)$ \\
\hline
\end{tabular}

Fonte: Autores (2021).

Já em relação ao gênero, pode-se observar superioridade de prevalência entre as pacientes do gênero feminino, se comparado ao gênero masculino, conforme evidenciado pela Tabela 3.

Estudos apontam prevalência de reações adversas em mulheres pelo fato de possuírem diferenças em aspectos fisiológicos e hormonais que interferem na farmacocinética e farmacodinâmica dos medicamentos aumentando a possibilidade de reações adversas (Rodrigues et al., 2020; Nagai et al., 2018).

Tabela 3. Gênero dos pacientes que apresentaram reações adversas detectadas no município de Cascavel - Pr, 2021.

\begin{tabular}{ll}
\hline Sexo & N $(\%)$ \\
\hline Feminino & $23(59)$ \\
Masculino & $16(41)$ \\
\hline
\end{tabular}

Fonte: Autores (2021).

Ao final do estudo, foram identificadas 39 RAMs, sendo que 19 (48\%) destes pacientes encontravam-se internados em unidades de terapia intensiva (UTI), 09 (23\%) em alas clínicas, 05 (13\%) em alas cirúrgicas, 04 (10\%) no centro cirúrgico e $02(6 \%)$ no pronto atendimento. O período de internamento em UTI 31,6 dias; na ala clínica 19,2; na ala cirúrgica 3,4 e os pacientes que apresentaram suspeitas de RAMs no centro cirúrgico a média de internação após a cirurgia foi de 3 dias. Não foi evidenciado associação das reações adversas com aumento do tempo de internamento.

Tabela 4. Gatilhos identificados e suas respectivas frequências no município de Cascavel - Pr, 2021.

\begin{tabular}{|c|c|}
\hline Gatilhos identificados & Frequência $(\%)$ \\
\hline Naloxona (Narcan®) & $0(0)$ \\
\hline Flumazenil (Lanexat ${ }^{\circledR}$ ) & $1(2)$ \\
\hline Metronidazol VO $\left(\right.$ Flagyl $\left.^{\circledR}\right)$ & $16(35)$ \\
\hline Dexclorfeniramina(Polaramine $\left.{ }^{\circledR}\right)$ & $3(7)$ \\
\hline Dexclorfeniramina+Betametasona (Celestamine $®)$ & $0(0)$ \\
\hline Fexofenadina (Allegra®) & $2(4)$ \\
\hline Hidroxizina (Hixizine®) & $1(2)$ \\
\hline Prometazina (Fenergan $®)$ & $13(28)$ \\
\hline Hidrocortisona (Solu-cortef®) & $10(22)$ \\
\hline Total & $46 *(100)$ \\
\hline
\end{tabular}

*Três reações adversas apresentaram mais de um medicamento gatilho.

Fonte: A autora (2021).

Foi possível identificar que a maior parte das suspeitas de RAMs ocorreu em pacientes internados em UTI, que são pacientes de maior complexidade submetidos a maior número de procedimentos e com farmacoterapia extensa, seguidos dos pacientes das alas clínicas que possuem maior complexibilidade do que os internados em áreas cirúrgicas, correlacionado com o tempo de internamento que foi maior nas alas clínicas (Gomes et al., 2020; Carvalho et al, 2013). Na Tabela 4, é possível observar a frequência dos gatilhos identificados durante o estudo. 
O gatilho mais utilizado foi metronidazol, sendo sua principal utilização o tratamento de colite pseudomembranosa que pode ser causada pelo uso de alguns antibióticos (Jesus, 2020). A colite pseudomembranosa é uma reação inflamatória intestinal ocasionada pelas toxinas de microrganismos oportunistas como $C$. difficile, que tem sua entrada no organismo facilitada quando há utilização de antibióticos de amplo-espectro (Fonteneles et al, 2020).

Os anti-histamínicos foram a classe de medicamento mais utilizada, sendo que um paciente com reação alérgica de pele fez uso de 4 medicamentos para controle dos sintomas (Prometazina, Hidrocortisona, Hidroxizina e Fexofenadina); um fez uso de 3 medicamentos (Fexofenadina, Hidrocortisona e Prometazina); e três fizeram uso de 2 medicamentos (Dexclorfeniramina e Prometazina). A Prometazina foi o anti-histamínico mais utilizado (13 pacientes), seguido da Dexclorfeniramina ( 3 pacientes), da Fexofenadina ( 2 pacientes) e Hidroxizina (1 paciente). Hidrocortisona foi prescrita para 10 pacientes.

A paciente que fez uso de 4 rastreadores era conhecidamente alérgica a penicilina, pode ter apresentado reação cruzada com Meropenem e Piperacilina/Tazobactam, por serem beta-lactâmicos. Apresentou melhora após suspensão da nutrição parenteral (NP), término dos antibióticos e da retirada de glúten da dieta via oral, que acabaram ocorrendo simultaneamente. Principais sinais e sintomas observados os demonstrados na Tabela 5.

Tabela 5. Principais reações adversas a medicamentos identificadas no município de Cascavel - Pr, 2021.

\begin{tabular}{lc}
\hline Sinais e sintomas & Frequência (\%) \\
\hline Diarreia & $16(41)$ \\
Potenciais indicadores de reação alérgica (prurido, eritema, hiperemia, rash & $22(56)$ \\
cutâneo, edema e urticária) & $1(3)$ \\
Sedação causada por benzodiazepínico & $0(0)$ \\
Sedação causada por opioides & \\
\hline
\end{tabular}

Fonte: Autores (2021).

O uso de antibióticos como: Ceftriaxona, Cefepime, Ceftazidima, Piperacilina/tazobactan, Meropenem, Azitromicina, Clindamicina e Teicoplanina, podem induzir colite e infecção por Clostridium difícile, sendo causa de 10-30\% de diarreia nosocomial infecciosa (Larentis, 2014). Para este estudo foi investigada se houve solicitação de exames para pesquisa de Clostridium difficile ou toxina desta bactéria, dos 16 pacientes que apresentaram diarreia após o uso de antimicrobiano apenas 6 tiveram exames solicitados para investigação de colite por Clostrodium, sendo que todos os resultados foram negativos (Casanova et al., 2019).

A administração de medicamentos e dietas via sondas enterais também podem ser causa de diarreia em pacientes internados (Fujino \& Nogueira, 2007). Estudos demonstram que as interações fármaco-nutrientes podem resultar na ineficácia dos medicamentos, além de alterar a biodisponibilidade dos nutrientes, o que pode alterar o estado nutricional bem com o estado geral do paciente (Morais et al., 2020; Kampa et al, 2020). Nessa pesquisa foram identificados 15 pacientes com diarreia que fizeram uso de alimentação via sonda. E destes, 13 tiveram medicamentos também administrados por esta via.

\section{Conclusão}

Apesar das RAMs terem sido identificadas e tratadas, o uso dos rastreadores foi útil para a investigação, notificação e registro nos prontuários dos pacientes, ficando acessível para consultas e possíveis internamentos posteriores. Embora a identificação da causalidade dessas reações seja difícil pela complexidade da farmacoterapia, o uso simultâneo de medicamentos horários padronizados e as reações adversas não ocorrerem imediatamente após a administração dos mesmos. 
Observou-se também que, pelo fato do setor de farmácia clínica do hospital possuir apenas uma profissional farmacêutica, houve dificuldades quanto a maior abrangência dos gatilhos, de maneira que foi necessário selecionar os principais rastreadores de relevância clínica. Neste contexto, vê-se a necessidade de aumentar o corpo clinico da farmácia hospitalar.

Adicionalmente, acreditamos que novos estudos envolvendo hospitais de médio ou grande porte seja necessário para que haja uma possível a comparação entre os resultados sugerindo a reprodutibilidade do método.

\section{Referências}

Almeida, C. M., \& Genaro, S. (2019). Cuidado na administração de medicamentos via sonda enteral. Colloquium Vitae, 11(3), 10-18. https://journal.unoeste.br/index.php/cv/article/view/2383.

Araujo, P. M., Braúna, C. C., Santos, V. R., Santana, L. C. L. R., Amorim, M. S. R., \& Coêlho, M. L. (2018). Avaliação dos resultados da metodologia de medicamentos gatilho para busca de reações adversas. Jornal de Ciências da Saúde, 1(3), 29-37. https://doi.org/10.26694/2595-0290.20181329-378493.

Araujo, A. W. M. S. (2019). Fatores de risco associado aos eventos adversos em pacientes internados com diagnóstico de sepse em um hospital de Sergipe: Um estudo de Coorte. Tese de Doutorado, Universidade Federal de Sergipe, Aracaju, SE, Brasil. http://ri.ufs.br/jspui/handle/riufs/13065.

Casanova, O., Penteado, S., \& Linartevichi, V. (2019). Análise de interações medicamentosas em unidade de terapia intensiva em um hospital no sul do Brasil. Fag Journal of Health, 1(1), 81-88. https://doi.org/10.35984/fjh.v1i1.6

Carvalho, D. C. M. F., Barbosa, L. M. G., Almeida, I. M., Cunha, C. H. M., \& Moreno, G. G. B. (2017). Manual de Farmácia Clínica e Cuidado ao Paciente. Atheneu.

Carvalho, R. E. F. L., Reis, A. M. M., Faria, L. M. P., Zago, K. S. A., \& Cassiani, S. H. B. (2013). Prevalência de interação medicamentos em unidades de terapia intensiva no Brasil. Belo Horizonte: Acta Paulista de Enfermagem, 26(2), 150-157. https://doi.org/10.1590/S0103-21002013000200008.

CFF. Conselho Federal de Farmácia. (2013). Resolução n ${ }^{\circ}$ 585, de 29 de agosto de 2013. https://www.cff.org.br/userfiles/file/resolucoes/585.pdf

Filho, J. M. (2014). Implantação de um serviço de farmacovigilância hospitalar e comparação dos algoritmos para análise de reação adversa a medicamento. Trabalho de Conclusão de Curso, Universidade Estadual Paulista, Araraquara, SP, Brasil. https://repositorio.unesp.br/ bitstream/handle/11449/110807/000792776_20191231.pdf.

Fonteneles, A. V., Vasconcelos, F. F., Santos, A. C \& Rocha, R. R. (2020). Colite pseudomembranosa causada pelo uso de antimicrobiano no âmbito hospitalar. Revista Multidisciplinar Em Saúde, 1(1), 26. https://editoraime.com.br/revistas/index.php/rems/article/view/101.

Fujino, V., Nogueira, L. A. B. N. S (2007). Terapia nutricional enteral em pacientes graves: revisão de literatura. Arquivo de Ciências da Saúde, 14(4), 220226. https://doi.org/10.5020/18061230.2017.p255.

Gallagher, R. M., Kirkham, J. J., Mason, J. R., Bird, K. A., Williamson, P. R., Nunn, A. J., Turner, M. A., Smyth, R. L. \& Pirmohamed, M. (2011). Development and inter-rater reliability of the Liverpool adverse drug reaction causality assessment tool. Plos One, 6(12), 28096. https://doi.org/10.1371/journal.pone.0028096.

Gomes, A. B. de S., Medeiros, F. R. F., \& Neto, M. P. L. (2020). Análise logística da assistência farmacêutica de um hospital do nordeste brasileiro. Research, Society and Development, 9(2), e155922210. https://doi.org/10.33448/rsd-v9i2.2210

Jesus, R. O. (2020). Tratamento de diarreia causada por antibiótico. Trabalho de Conclusão de Curso, Faculdade Maria Milza, Governador Mangabeira, BA, Brasil.

Kampa, J. C. C., Reis, L. O., Mezzoomo, T. R \& Camargo, C. Q. (2020). Pacientes sob terapia nutricional enteral e prevalência de interação fármaconutrientes no ambiente hospitalar. Research, Society and Development, 9(3), 1-6. http://dx.doi.org/10.33448/rsd-v9i3.2680.

Larentis, D. Z. (2014). Fatores de risco e prognóstico associados à diarreia por Clostridium difficile em pacientes adultos hospitalizados. Dissertação de Mestrado, Universidade Federal do Rio Grande do Sul, Porto Alegre, RS, Brasil.

Minella, F. C. O., \& Linartevichi, V. F. (2021). Effects of cannabidiol on the signs and comorbidities of autistic spectrum disorder. Research, Society and Development, 10(10), e64101018607. https://doi.org/10.33448/rsd-v10i10.18607

Morais, I. T. de S., Torres, N. M. F., Martins, J. A., Santana, L. S. O. S., Veloso, V. L., Santos, L. C. R., Valente, R. de C., Oliveira, B. E. da S., Alves, A. K. de S., Castro, R. F. de, Aquino, S. leão de, Abreu, F. L. de, \& Moura, M. C. L. de. (2020). Análise clínica de prescrições de antibióticos em um hospital privado em Teresina - Piauí, Brasil. Research, Society and Development, 9(7), e451974401. https://doi.org/10.33448/rsd-v9i7.4401

Nagai, K. L., Takahashi, P. S. K., Pinto, L. M. O., \& Romano-Lieber. (2018). Uso de rastreadores para busca de reações adversas a medicamentos como motivo de admissão de idosos em pronto-socorro. Ciência \& Saúde Coletiva, 23(11), 3997-4006. https://doi.org/10.1590/1413-812320182311.27022016.

OMS. Organização Mundial de Saúde. (2005). Segurança dos medicamentos: um guia para detectar e notificar reações adversas a medicamentos. Brasília, OPAS.

OPAS. Organização Pan-Americana de Saúde. (2002). Consenso brasileiro de atenção farmacêutica: proposta. Brasília, OPAS. https://bvsms.saude.gov.br/bvs/publicacoes/PropostaConsensoAtenfar.pdf. 
Research, Society and Development, v. 10, n. 13, e392101321367, 2021

(CC BY 4.0) | ISSN 2525-3409 | DOI: http://dx.doi.org/10.33448/rsd-v10i13.21367

Rodrigues, H. S., Souza, R. P., Sousa, R. W. R. de, Pereira, L. C. A., Branco, R. R. de O. C., Silveira, P. H. F. P., \& Britto, M. H. R. M. (2020). Perfil de interações medicamentosas de Agentes Antineoplásicos Orais (AAOs) dispensados para pacientes oncológicos. Research, Society and Development, 9(8), e145985369. https://doi.org/10.33448/rsd-v9i8.5369

Souza, L. B., Souza, D. M., Souza, S. M., Silva, D. R. \& Aguilar, N. C. (2018). Importância do farmacêutico clínico no uso seguro e racional de medicamentos no âmbito hospitalar. Pensar Acadêmico, 16(1), 24. https://doi.org/10.21576/rpa.2018v16i1.360.

Sousa, C. J. P., Prete, A. C. L. \& Ribeiro, C. H. M. A. (2020). Acurácia de gatilhos na identificação de eventos adversos a medicamentos em idosos hospitalizados. Research, Society and Development, 9(12), 1-28. http://dx.doi.org/10.33448/rsd-v9i12.11135.

Sousa, C. J. P., Prete, A. C. L., Gomes, A. G. P., Castro, E. F. R. \& Ribeiro, C. H. M. A. (2021). Adverse drug events in hospitalized patients in Brazil: Integrative literature review. Research, Society and Development, 10(4), 1-13. https://doi.org/10.33448/rsd-v10i4.13818.

Souza, C. M. (2016). Incidência, caracterização e acompanhamento da resolução de eventos adversos aos medicamentos em pacientes admitidos em emergência pediátrica. Dissertação de Mestrado, Universidade Estadual de Campinas, Campinas, SP, Brasil. http://repositorio.unicamp.br/jspui/handle/REPOSIP/321094. 